\title{
Medical Residents and Interprofessional Interactions in Discharge: An Ethnographic Exploration of Factors That Affect Negotiation
}

\author{
Joanne Goldman, $P h D^{1,2}$, scott Reeves, $P h D^{3}$, Robert Wu, MD ${ }^{4,5}$, Ivan Silver, MD, MEd \\ Kathleen MacMillan, $P h D, R N^{7}$, and Simon Kitto, $P h D^{8}$
}

${ }^{1}$ Institute of Medical Sciences, Faculty of Medicine, University of Toronto, Toronto, ON, Canada; ${ }^{2}$ Wilson Centre, University Health Network, Toronto, ON, Canada; ${ }^{3}$ Center for Health \& Social Care Research, Faculty of Health, Social Care \& Education, Kingston University \& St George's, University of London, London, UK; ${ }^{4}$ Department of Medicine, Faculty of Medicine, University of Toronto, Toronto, ON, Canada; ${ }^{5}$ Division of General Internal Medicine, Toronto General Hospital, University Health Network, Toronto, ON, Canada; ${ }^{6}$ Centre for Addiction and Mental Health, Department of Psychiatry, Faculty of Medicine, University of Toronto, Toronto, ON, Canada; ${ }^{7}$ School of Nursing, Dalhousie University, Halifax, NS, Canada;

${ }^{8}$ Department of Innovation in Medical Education, Faculty of Medicine, University of Ottawa, Ottawa, ON, Canada.

BACKGROUND: Interprofessional collaboration is an important aspect of patient discharge from a general internal medicine (GIM) unit. However, there has been minimal empirical or theoretical research that has examined interactions that occur between medical residents and other healthcare professionals in the discharge process. This study provides insight into the social processes that shape and characterize such interactions.

OBJECTIVE: To explore factors that shape interactions between medical residents and other healthcare professionals in relation to patient discharge, and to examine the opportunities for negotiations about discharge between these professional groups.

DESIGN: A qualitative ethnographic approach using observations, interviews and documentary analysis.

PARTICIPANTS AND SETTING: Healthcare professionals working in a GIM unit in Canada.

APPROACH: Sixty-five hours of observations were undertaken in a range of settings (e.g. interprofessional rounds, medical and nursing rounds, nursing station) in the unit over a 17-month period. A maximum variation sampling approach was used to identify healthcare professionals working in the unit. Twenty-three interviews were completed, recorded and transcribed verbatim. A directed content approach using theories of medical dominance and negotiated order was used to analyze the data.

KEY RESULTS: The organization of clinical work in combination with clinical teaching influenced interprofessional interactions and the quality of discharge in this GIM unit. While organizational activities (orientation and rounds) and individual activities (e.g. role modeling, teaching) supported negotiations between medical residents and other healthcare professionals around discharge, participants had varied perspectives about their effectiveness.

CONCLUSIONS: This study illuminates social factors and processes that require attention in order to address challenges with interprofessional collaboration and discharge

Received June 27, 2014

Revised November 24, 2014

Accepted March 17, 2014

Published online April 14, 2015 in GIM. These findings have implications for medical education, workplace learning, patient safety and quality improvement.

KEY WORDS: Medical education-practice-based learning; Qualitative research; Quality improvement; Health services research.

J Gen Intern Med 30(10): 1454-60

DOI: $10.1007 / \mathrm{s} 11606-015-3306-6$

(C) Society of General Internal Medicine 2015

\section{INTRODUCTION}

Safe and timely patient discharge from the hospital is critical, particularly in general internal medicine (GIM), given the unplanned nature of patient admissions, range and complexity of patient issues, the varied types healthcare professionals providing care, and annual increases in patient volume. ${ }^{1-3}$ Government and hospital policies identify the importance of interprofessional collaboration for patient discharge, yet research findings report challenges with such practice. ${ }^{4-10}$ Physicians in GIM have reported diverse discharge interprofessional experiences. Some described their interprofessional 'teams' as fragmented and characterized by last-minute problemsolving, whereas others were described as cohesive. ${ }^{7}$ Problems with communication in discharge processes have been attributed to professional hierarchy, insufficient contact among healthcare professionals, and lack of clarity about professional roles and responsibilities. ${ }^{7-10}$

Initial research has explored "teamwork" in hospital discharge $^{7-10}$, but there has been less critical examination of the complex range of factors that affect the ways in which healthcare professionals work together (e.g. interprofessional friction, role boundaries, miscommunication, poor coordination of care $)^{11}$ in GIM from the perspective of the various healthcare professionals. We therefore undertook a sociologically informed ethnographic study to examine the factors that shape and characterize such interactions in relation to the process of discharge. ${ }^{12,13}$ Interprofessional collaboration has been defined as a type of work which involves different 
healthcare professionals who regularly come together to solve problems or provide services. ${ }^{11}$

We used two sociological theories to inform the study: professional dominance and negotiated order. The theory of professional dominance is concerned with the nature and implications of healthcare division of labour, where physicians have historically occupied a dominant clinical, economic and social position over other occupational groups. ${ }^{14,15}$ Negotiated order theory focuses on micro-level interactions and offers insight into how individuals interact within, and in resistance to, factors such as healthcare division of labour. ${ }^{16}$ According to Strauss, negotiation involves "the continual working out together of who [is] to do what, how and with whom" (p. 107).

The focus of this paper on interactions between medical residents and other healthcare professionals in relation to discharge is timely given the ongoing debate about the tensions between education and quality of care in practice ${ }^{17,18}$ and recommendations for medical education to address teambased care, patient safety and quality improvement ${ }^{17,19}$.

\section{METHODS}

\section{Study Design and Setting}

We used an ethnographic approach in this study. ${ }^{13,20}$ Ethnography is concerned with the everyday experiences of individuals, organizations, and society, with a commitment to understanding the cultural context in which these experiences and social interactions take place. ${ }^{21}$ We collected information in the form of observational, interview and documentary data from January 2012 to May 2013. We report mainly on interview data, although the analysis was informed by observation and documentary data as well. We received ethics approval for this study from the hospital research ethics board and affiliated university.

The study was conducted in the GIM unit of an academic teaching hospital in Canada. This unit provides care for patients with complicated medical problems and follow-up for patients discharged from emergency departments and medical and surgical wards. The unit has approximately 50-70 nurses, 16 medical residents, 12 attending physicians, four social workers, pharmacists and community care access centre case managers, three physiotherapists and occupational therapists, and a speech-language pathologist, dietitian and spiritual care worker. The ward is a designated clinical teaching unit (CTU), and is composed of four medical teams and a fifth hospitalist team. Each medical team includes one attending physician and senior resident, two or more junior residents, and two to four students. A social worker, physiotherapist, occupational therapist and pharmacist are aligned with each team, and the other healthcare professionals work with all of the teams. The hospital patient flow and utilization-of-care coordinators work with all hospital units. Structured interprofessional care rounds organized to improve patient flow and discharge planning occur weekday mornings.
In ethnography, the initial interests and questions underlying the research are refined over time as data collection and analysis occur simultaneously. ${ }^{20}$ Our aim was to explore interprofessional interactions in relation to the discharge process; one theme that emerged - and we consequently pursued in data collection and analysis, and report here-was the impact of a clinical teaching unit on these interactions.

\section{Sampling Strategy}

The first author (JG) undertook nonparticipant observations and conducted one-on-one semi-structured interviews. JG observed a range of activities and settings within the GIM unit, including morning rounds, nursing stations, medical rounds, nursing rounds, and orientations; in addition, she job-shadowed a pharmacist, physiotherapist and nurse. Observations focused on conversations and interactions linked to discharge activities. JG conducted observations at different times during the weekday from 7:00 am to 4:30 pm, when staff across the complete range of healthcare professionals were most likely working in the unit and activity concerning discharge was highest. During observations, JG took fieldnotes about people, spaces, objects, actions and interactions, in addition to conversation that was occurring $^{20,21}$ that was focused on discharge.

For the interviews, we used a maximum variation sampling approach $^{22}$ with the aim of interviewing individuals representing each professional group working in GIM. Interview participants were purposively selected from the staff directory based on their professional group and on recommendations from participants. All but two professionals who were approached agreed to participate in an interview. Data collection stopped when we had determined that the perspectives of the range of healthcare professionals were represented in the interview data, and that the various spaces in the GIM unit relevant to patient discharge discussions and interactions had been observed.

\section{Data Collection}

JG wrote fieldnotes during the observations on the GIM unit, and later typed these notes in greater detail, including verbatim reports, a summary of dialogue and behaviours, reactions to the data, and interpretations. ${ }^{20,21}$ In total, she collected approximately 65 hours of observations. We developed the interview guide based on initial observations and the interprofessional collaboration and discharge literature. The basic interview guide is included in Appendix A. As is typical of ethnography ${ }^{20}$, the guide was adapted to pursue emerging areas and the professional background of the participant. Interviews were audio-recorded and transcribed verbatim. Interview participants received a $\$ 20$ gift card. In addition to observations, JG gathered documentary data in the form of publicly available (online) provincial government and hospital documentation regarding discharge. These data were used to explore the connections and/or disconnections between policies on discharge and actual practice, and informed data collection in observations and interviews. 


\section{Data Analysis}

We conducted data collection and analysis iteratively, and explored emerging themes. ${ }^{20,21}$ We coded the data using a directed content approach, whereby analysis begins with the selection of a relevant theory to help generate the initial codes, and then aims to refine and extend the theory. ${ }^{23}$ As noted above, the theories of professional dominance and negotiated order $^{14-16}$ informed coding and analysis.

Data, method and theory triangulation allowed for the development of a more comprehensive understanding of the phenomena being studied. ${ }^{24,25}$ Firstly, we collected observational data in different settings and with representatives from different healthcare professional groups. Secondly, we compared data from observations, interviews and discharge documents. Thirdly, we used the two theories mentioned above to explore the data from their different perspectives. Reflexivity was supported in multiple ways. A structured fieldnote approach allowed for descriptive, interpretive and reflective notes. Preliminary findings were presented at conferences to obtain stakeholders' input. We also discussed team members' interpretations of data based on professional and theoretical positions.

The first author was a doctorate student specializing in medical sociology and interprofessional interactions, with no prior connections to this clinical unit. The coauthors acted as supervisors and committee members who provided guidance on the research project. All authors met regularly to review data collection, coding and emerging themes.

\section{RESULTS}

Twenty-three interviews were conducted. Participants comprised five nurses; three attending physicians; two medical residents, social workers, and patient flow and utilization-ofcare coordinators; and one physiotherapist, occupational therapist, pharmacist, speech language pathologist, community care access centre case manager, and spiritual care worker. Interviews ranged from 20 to 55 minutes. The results are presented in two sections. The first section describes the impact of the organization of work and clinical teaching on interprofessional interactions and the process of discharge. The second section reports on the nature of activities undertaken to support negotiations of interprofessional interactions regarding discharge.

\section{Organization of Clinical Work, Teaching and Discharge}

In this unit, physicians had the primary responsibility for discharge decision-making and summaries. This division of labour had a profound effect on the construction of the character and quality of interprofessional negotiations around discharge identified in this study. The medical trainees were expected to undertake these discharge responsibilities, learning "on the go", under the guidance of their medical attending.
The involvement of other professionals in the discharge process, though, resulted in the intersection of medical training, interprofessional interactions, and the discharge process, which affected the quality of discharge. Key issues included the rotational nature of residents' training, resident attitudes and knowledge regarding the roles of other healthcare professionals, and tensions between education and practice.

Medical trainees and healthcare professionals described the impact of the rotational nature of medical residents on the process of discharge. As one social worker commented:

"It's by no means their fault that they don't know you have to do xyz to have this discharge happen...I know they're going to make that mistake, it always happens, and it would be nice for it not to happen, because as soon as you spend two months teaching them and they learn it and they totally get it, they're gone, and you get a new batch..." (Social worker, Interview \#5)

Medical residents explained that it takes time to develop relationships with the healthcare professionals and to understand, for example, details of form completion and signatures.

According to healthcare professionals, particularly those in social work, physiotherapy, occupational therapy and pharmacy, the medical residents' knowledge of and attitudes towards their roles in discharge affected discharge practices. These professionals noted that the first week of a rotation was usually problematic for over- and under-referring. For example, one concern involved the fact that physiotherapists did not need to see patients who were at their baseline, but that residents did not appreciate the notion of "baseline" until halfway through their rotation. The pharmacists commented that their extensive role in the discharge process was often not appreciated by residents. For instance, residents would discharge a patient without the pharmacist's knowledge. Pharmacists sometimes felt like they had to act as "discharge police" at the beginning of a rotation due to a lack of communication. The social workers described having to deal with residents' mistakes with discharge plans, such as failing to consult with them and making recommendations inappropriately. As a social worker stated:

"I'm constantly saying to residents... that's not the discharge plan, that's wrong; but then if I don't correct them, then the nurses hear the wrong plan and the wrong plan then gets reinforced to the family, and it's like a bad cycle..." (Social worker, Interview \#21)

The social workers, physiotherapists, occupational therapists and pharmacists recognized that it takes time for medical trainees to understand their roles in discharge. Some noted variability, however, in the degree to which medical trainees were open to involvement:

"So maybe my residents and medical students are super great with medications...or very pro-pharmacy, 
and ask me a lot of questions...so you know that may be a good team to me, right? The social worker thinks, oh, they're really compassionate... it does vary quite a bit..." (Pharmacist, Interview \#15)

Many of the interviewees expressed their enjoyment with working in a teaching hospital, yet they also reported concerns about tensions between education and quality of care, noting that at times the focus on medical training was overemphasized at the expense of care, such as an interprofessional approach to discharge. Some of them attributed medical residents' behaviours to the pressure of being a trainee, and being expected to have an answer for the medical attending despite their limited knowledge of discharge policies. As a senior resident commented, the responsibility for discharge could be particularly challenging for medical students and junior residents, as

"...they are often overwhelmed by the active medical issues [and do not yet appreciate the need to] think about the next step...the whole path for this patient" (Senior resident, Interview \#14).

\section{Negotiations: Interprofessional Interactions and Discharge}

Given the nature of medical responsibility for discharge and the involvement of other healthcare professionals in the discharge process, as described in the previous section, activities to support ongoing interprofessional negotiations were needed. In this section we report on activities undertaken to support these negotiations, including variation in perceptions of their function, practice and effectiveness. In addition, we present findings concerning professionals' strategies for circumventing existing arrangements when the need arose.

Unit Activities. Two GIM unit activities aimed to support negotiations between medical residents and other healthcare professionals in discharge: orientation for medical residents and interprofessional morning care rounds.

In an orientation at the beginning of each medical rotation, all of the healthcare professionals, with the exception of nurses, explained their roles, and when and how to refer to and consult with them. Although physicians and other professionals expressed concerns that this orientation was merely a ritual that residents endured while anxiously waiting to begin their work, healthcare professionals also emphasized the value of this orientation:

“...the times that I haven't been able to participate in the...orientation, I've definitely seen a difference in terms of even just knowing...who am I looking for, what faces am I looking for, and them recognizing me as part of their team as well, and then knowing a little bit about what I do at least..." (Occupational therapist, Interview \#13)
Some professionals reported feeling frustrated when medical attendings minimized the importance of the orientation or excused their medical residents from attending.

Morning care rounds largely focused on discharge planning, and medical residents were expected to lead the discussion about their designated patients. The physicians expressed different perceptions about the function of these rounds in the education and practice of interprofessional interactions and patient discharge. From one perspective, rounds were viewed as a ritual where education was not an explicit aim; rather, they were a place where the healthcare professionals instructed medical residents how to function such as which forms to complete for discharge. The other perspective held that residents "learn by doing", and that their participation in rounds enabled them to learn how to interact with other healthcare professionals and about their roles and contributions to discharge.

While both professionals and medical residents described the importance of interprofessional interactions during rounds, the format of rounds could also be a source of frustration, given the short time frame and the large number of GIM workers involved, each with different priorities. Furthermore, the priority to support medical residents' professional development created challenges at times for healthcare professionals' goals of patient care, as rounds were the only time during the day that they met formally to discuss discharge.

Individual Activities. Many professionals described individual-level initiatives undertaken to support negotiations between healthcare professionals and medical residents. These activities were performed at the discretion of the medical attendings and other healthcare professionals, and therefore varied in practice.

The attendings and many of the other professionals described how medical residents learned from their senior medical colleagues how to relate to, and communicate with, other professionals. Variation was reported among medical attendings in the extent to which they formally and informally taught interprofessionalism around discharge processes. Physician role modeling and guidance concerning the role of other healthcare professionals in discharge influenced medical residents' approaches to interprofessional interactions. The following fieldnote is an example of an attending providing guidance during medical rounds:

Resident: Perhaps OT, PT to see once better.

Staff: She's from home, fairly highly function; does she have supports?

Resident: I didn't ask.

Staff: Admitted with some belly pain, might not need much support from allied team if highly functional. Have you already written order?

Resident: No.

Staff: Let's hold off, waste for PT to do big assessment if going home, likely going home on weekend.

(Fieldnote) 
The other professionals, such as nurses, physiotherapists and social workers, perceived that such behaviours impacted on the appropriateness of interprofessional interactions about discharge and, therefore, the efficiency of patient discharge. For example, a social worker explained that failure to consult could affect the timeliness of patient discharge, which in turn affected patient flow:

“...the residents hear, oh, ask social work this, ask physio this, ask OT, and so then they're learning through experience from the top, whereas there's a few people that don't do that, and you can tell the difference $100 \%$ in terms of the numbers (of patients) go up..." (Social worker, Interview \#21)

Certain attendings organized informal social get-togethers to support medical residents and interprofessional interactions. In addition, a couple of participants recalled a medical attending who had organized weekly formal teaching sessions where other professionals took turns teaching medical trainees about their areas of expertise, although these were not occurring at the time of the interview.

The social workers, physiotherapists, occupational therapists and pharmacists described their efforts to support interprofessional interactions around discharge. For example, a pharmacist commented that she aimed to teach medical residents to do discharge reconciliation rather than doing it for them and having them sign off. One social worker stated that she made herself accessible, distributed handouts of discharge plans, and sent messages to the medical trainees:

"I did a check-in with my med students last week...do you guys have any questions, like how are you finding it...do you know what social work does, do you want to run the list...they were really receptive...both of them are, like, I still don't really know what you do." (Social worker, Interview \#21)

These professionals also expressed a need for more systematic opportunities to provide education about their areas of expertise in discharge to the medical residents. For example, a pharmacist commented that it would be ideal to have a set time at the beginning of each rotation to teach the residents how to discharge and what to consider, such as medications covered by formulary.

The professionals explained that when negotiations with medical residents about discharge were not productive, they circumvented the expectation to work with medical residents, and instead communicated directly with the medical attending to ensure that information was clearly communicated:

“...if the staff (attending physician) is around we tend to... touch base with the staff rather than the residents or medical students, because we do know them, and know that ultimately the decisions come from them and they like to be in the loop. Sometimes I don't know whether or not, if I tell the resident or the med student, if that message gets relayed on to the staff." (Occupational therapist, Interview \#13)

\section{DISCUSSION}

Using the theories of medical dominance and negotiated order, these findings suggest that medical responsibility for discharge and the presence of clinical teaching activities intersect with an interprofessional approach to discharge to affect the process and quality of discharge. Although orientation and rounds and individual teaching activities are undertaken to support negotiations, perceptions of their practice and effectiveness are varied. The findings raise important questions about clinical training and medical responsibility, structural factors that are frequently taken for granted as natural and fixed rather than normative and variable. Furthermore, they provide direction for supporting negotiations between medical residents and other healthcare professionals in discharge.

This study reinforces the need for ongoing attention to the balance between medical education and patient care, a topic identified as an area of priority in medical education research. ${ }^{18}$ Strategies to address this balance include further attention to education about discharge and alternative interprofessional approaches for discharge, particularly given the turnover of medical attendings and residents. Greysen et al. ${ }^{26}$ outline several ways to improve residency training in discharge, such as the development of educational goals and activities as part of the formal curriculum; incorporating key concepts embedded in high-quality discharge, such as interprofessional care, patient safety and patient communication, into broader themes of systems-based practice training; and the use of innovative approaches to evaluation. In addition to education, various interprofessional arrangements for discharge are being examined, such as embedding a discharge facilitator in a resident team. ${ }^{27}$ Planning and assessment of such interventions requires attention to the complex issues concerning role changes, professional boundaries and patient care. ${ }^{28}$

Given the organization of this GIM unit, activities were undertaken to support negotiations between medical trainees and other professionals in relation to discharge. The mixed perceptions of the value of the orientation might be indicative of the lack of consistent support by all stakeholders and positioning within a broader planned interprofessional education and practice strategy. A more explicit approach would consider needs and recommendations across the range of stakeholders in supporting teaching and practice of discharge, such as the pharmacist in this study who described the value of having an opportunity to teach residents about discharge. Such an approach could identify strategies such as an orientation that, in combination with other formal and informal approaches, would reflect evidence-based developments in interprofessional education that optimize learning outcomes. ${ }^{29,30}$ 
Research on rounds in other contexts has demonstrated disjunction between teaching valued by medical attendings and learning valued by trainees, ${ }^{31,32}$ and disconnections between medical and other healthcare professionals regarding the effectiveness of rounds in meeting education and patient care goals. ${ }^{33}$ This study encourages further consideration of the objectives and processes of education practices such as orientations and rounds. Such reflexivity can contribute to a greater understanding of objectives that are and are not being met and the implications of these practices. ${ }^{34}$

In the GIM unit in this study, teaching regarding discharge processes and interprofessional interactions was dependent on initiatives by individual physicians and other healthcare professionals. This finding reflects researchers' calls to include other healthcare professionals in educating residents and providing feedback on their performance, and the need for medical staff training in relation to discharge processes. ${ }^{3,26}$ While individual efforts are laudable, it is important to create environments conducive to interprofessional interactions and to maximize the contributions of different professionals to care rather than relying on the benevolence of individuals. ${ }^{35}$ The creation of an effective workplace learning culture was identified as another priority area in a survey of medical education research. ${ }^{18}$ In this survey, workplace learning was conceptualized as balancing training and service conflicts and creating an effective workplace learning culture. These ideas reflect workplace learning as a process that supports desirable outcomes in the context of organizational goals and individual career development. ${ }^{36}$ Workplace learning would ideally maximize teaching opportunities around discharge while considering and supporting quality patient care.

The present study was not without limitations. The findings are based on one GIM unit in Canada, and the organization of CTUs, professionals, and GIM units may differ in other hospitals. While our sample of 23 interviews might appear small, however, these data were complemented and compared with observational data. Furthermore, the theoretical sampling approach aimed to ensure variation in professional representation, given the goal of examining interprofessional interactions. The findings from this study are important from the standpoint of conceptual rather than empirical generalizability. ${ }^{25}$ Conceptual generalizability refers to the ability of findings inform healthcare contexts that differ from that in which the original study was undertaken. This study adds a Canadian perspective to the largely American and British qualitative research ${ }^{7,9,10,26}$ on discharge and interprofessional interactions. The reporting of similar themes across studies allows for strengthening of the evidence while simultaneously demonstrating international relevance of the findings.

\section{CONCLUSIONS}

This study illuminates the factors and processes that require attention in order to address challenges with interprofessional collaboration within the discharge process in GIM. The use of professional dominance has provided a lens through which to examine how medical responsibility for discharge, including medical trainees' responsibilities, affects interactions between medical residents and other professionals and the quality of discharge. In addition, negotiated order theory has provided a means to explore both the opportunities for interprofessional negotiations and the limitations of these opportunities. Efforts to improve discharge should be mindful of both theoretical perspectives, thus attending to the complex sociocultural context of the GIM unit. The findings from this study can inform future developments in medical education and workplace learning to optimize patient safety and quality of care.

Acknowledgments: This research was conducted as part of Joanne Goldman's doctoral dissertation, which was funded by the Canadian Institutes of Health Research. Joanne was the recipient of a Frederick Banting and Charles Best Canada Graduate Scholarship Doctoral Award. We would like to thank the GIM staff that participated in the interviews and observations in this research.

Conflict of Interest: The authors declare that they do not have a conflict of interest.

Corresponding Author: Joanne Goldman, PhD; Wilson CentreUniversity Health Network, Toronto, ON, Canada (e-mail: joanne.goldman@utoronto.ca).

\section{REFERENCES}

1. Szecket N, Wong HJ, Wu RC, Berman HD, Morra D. Implementation of a continuous admission model reduces the length of stay of patients on an internal medicine clinical teaching unit. J Hosp Med. 2012;7:55-9.

2. Wong HJ, Morra D, Caesar M, Carter MW, Abrams H. Understanding hospital and emergency department congestion: an examination of inpatient admission trends and bed resources. CJEM. 2010;12:18-26.

3. Davis MM, Devoe M, Kansagara D, Nicolaidis C, Englander H. Did I do as best as the system would let me? Healthcare professional views on hospital to home care transitions. J Gen Intern Med. 2012;27:1649-56.

4. Bryan K. Policies for reducing delayed discharge from hospital. BMJ. 2010;95:33-46.

5. Ontario Hospital Association. Achieving Patient Experience Excellence in Ontario: An Idea Book. 2013 Spring. Available at: http://www.oha.com/ CurrentIssues / keyinitiatives / Patient\%20Experience/Documents / Final\%20-\%20Idea\%20book.pdf. Accessed March 2, 2015.

6. Office of the Auditor General of Ontario. 2010 Annual report. 2010. Available at: http://www.auditor.on.ca/en/reports_en/en10/2010ar_en. pdf. Accessed March 2, 2015.

7. Greysen SR, Schiliro D, Horwitz LI, Curry L, Bradley EH. "Out of sight, out of mind": housestaff perceptions of quality-limiting factors in discharge care at teaching hospitals. J Hosp Med. 2012;7:376-81.

8. Wong ELY, Yam CHK, Cheung AWL, et al. Barriers to effective discharge planning: a qualitative study investigating the perspectives of frontline healthcare professionals. BMC Health Serv Res. 2011;11:242.

9. Connolly M, Grimshaw J, Dodd M, et al. Systems and people under pressure: the discharge process in an acute hospital. J Clin Nurs. 2009;18:549-58.

10. Pethybridge J. How team working influences discharge planning from hospital: a study of four multi-disciplinary teams in an acute hospital in England. J Interprof Care. 2004; 18:29-41.

11. Reeves S, Lewin S, Espin S, Zwarenstein M. Interprofessional Teamwork for Health and Social Care. United Kingdom: Wiley-Blackwell; 2010.

12. Kitto S, Chesters J, Thistlethwaite J, Reeves S. Sociology of Interprofessional Health Care Practice. New York: Nova Science Publishers, Inc.; 2011.

13. Reeves S, Peller J, Goldman J, Kitto S. Ethnography in qualitative educational research: AMEE Guide No. 80. Med Teach. 2013;35:E1365-E79.

14. Freidson E. Profession of Medicine: A Study of the Sociology of Applied Knowledge. Chicago: The University of Chicago Press; 1988. 
15. Willis E. Medical Dominance: The Division of Labour in Australian Health Care. Sydney: Allen \& Unwin; 1989.

16. Strauss AL. Negotiations: Varieties, Contexts, Processes and Social Order. London: Jossey-Bass; 1978.

17. Chang A, Fernandez H, Cayea D, et al. Complexity in graduate medical education: a collaborative education agenda for internal medicine and geriatric medicine. J Gen Intern Med. 2014;29:940-6.

18. Dennis AA, Cleland JA, Johnston P, Ker JS, Lough M, Rees CE. Exploring stakeholders' views of medical education research priorities: a national survey. Med Educ. 2014;48:1078-1091.

19. Wong BM, Etchells EE, Kuper A, Levinson W, Shojania KG. Teaching quality improvement and patient safety to trainees: a systematic review. Acad Med. 2010;85(9):1425-39.

20. Hammersley M, Atkinson P. Ethnography: Principles in Practice. 3rd ed. London: Routledge; 2007.

21. Emerson RM, Fretz RI, Shaw LL. Writing Ethnographic Fieldnotes. 2nd ed. Chicago: University of Chicago Press; 2011.

22. Patton MQ. Qualitative Research and Evaluation Methods. 3rd ed. Thousand Oaks, CA: Sage Publications, Inc.; 2002.

23. Hsieh HF, Shannon SE. Three approaches to qualitative content analysis. Qual Health Res. 2005; 15:1277-88.

24. Flick U. Triangulation revisited: strategy of validation or alternative? J Theory Soc Behav. 1992;22:175-97.

25. Kitto SC, Chesters J, Grbich C. Quality in qualitative research. MJA. 2008;188:243-6.

26. Greysen SR, Schiliro D, Curry L, Bradley EH, Horwitz LI. "Learning by Doing"-Resident perspectives on developing competency in high-quality discharge care. J Gen Intern Med. 2012;27:1188-94.

27. Finn KM, Heffner R, Chang $\mathbf{Y}$, et al. Improving the discharge process by embedding a discharge facilitator in a resident team. $\mathrm{J}$ Hosp Med. 2011;6:494-500.

28. Powell AE, Davies HTO. The struggle to improve patient care in the face of professional boundaries. Soc Sci Med. 2012;75:807-14.

29. Reeves S, Tassone $\mathbf{M}, \mathbf{P a r k e r} \mathbf{K}$, et al. Interprofessional education: an overview of key developments in the past three decades. Work. 2012;41:233-45.

30. Varpio L, Bidlake E, Casimiro L, et al. Resident experiences of informal education: how often, from whom, about what and how. Med Educ. 2014;48:1220-34.

31. Kuper A, Zur Nedden N, Etchells E, Shadowitz S, Reeves S. Teaching and learning in morbidity and mortality rounds: an ethnographic study. Med Educ. 2010;44:559-69.

32. Walton JM, Steinert Y. Patterns of interaction during rounds: implications for work-based learning. Med Educ. 2010;44:550-8.

33. Birtwistle L, Houghton JM, Rostill H. A review of surgical ward round in a large paediatric hospital: does it achieve its aims? Med Educ. 2000;34:398-403.
34. Carroll K, Iedema R, Kerridge R. Reshaping ICU ward round practices using video-reflexive ethnography. Qual Health Res. 2008;18:380-90.

35. Nugus P, Greenfield D, Travaglia J, Westbrook J, Braithwaite J. How and where clinicians exercise power: interprofessional relations in health care. Soc Sci Med. 2010;71:898-909.

36. Matthews P. Workplace learning: developing an holistic model. Learn Organ. 1999;6:18-29.

\section{APPENDIX A: INTERVIEW GUIDE}

1. Can you describe your role as a XXX (e.g. social worker, nurse, etc.) in GIM?

2. What is your role in relation to discharge in GIM?

3. What activities do you undertake in patient discharge?

4. Who do you interact with in relation to discharge decisions in GIM?

5. What is your perception of other people's roles in relation to discharge?

6. How do hospital procedures and policies influence your activities related to discharge?

7. What is the nature of your interactions with patients concerning discharge?

8. Do you think there are patient safety issues in relation to discharge? If yes, what are they? What is a 'safe' discharge?

9. What are your perceptions of communication concerning patients and discharge? Where does this communication happen? How? Are there limitations?

10. Do you use the electronic whiteboard? How?

11. Where do discussions and decisions about discharge happen?

12. Who is involved in family meetings about discharge?

13. Can you describe a case that was a 'good discharge' or a 'bad discharge'?

14. What is your perception of readmissions? 